\title{
Measure and Dimension of Solenoidal Attractors of One Dimensional Dynamical Systems
}

\author{
A. M. Blokh and M. Yu. Lyubich
}

Steklov Mathematical Institute, LOMI, Fontanka 27, SU-191011 Leningrad, USSR

\begin{abstract}
Let $f: M \rightarrow M$ be a $C^{\infty}$-map of the interval or the circle with non-flat critical points. A closed invariant subset $A \subset M$ is called a solenoidal attractor of $f$ if it has the following structure: $A=\bigcap_{n=1}^{\infty} \bigcup_{k=0}^{p_{n}-1} I_{k}^{(n)}$, where $\left\{I_{k}^{(n)}\right\}_{k=0}^{p_{n}}$ is the cycle of intervals of period $p_{n} \rightarrow \infty$. We prove that the Lebesgue measure of $A$ is equal to zero and if $\sup \left(p_{n+1} / p_{n}\right)<\infty$ then the Hausdorff dimension of $A$ is strictly less than 1 .
\end{abstract}

\section{Introduction}

Let $M$ be a one dimensional compact manifold with boundary, i.e. a finite union of disjoint intervals and circles. Let us consider the class $\mathfrak{A}$ of $C^{\infty}$-smooth transformations $f: M \rightarrow M$ with non-flat critical points [the last means that for each critical point $c$ there exists $n$ such that $\left.f^{(n)}(c) \neq 0\right]$. The map $f$ is called $d$-modal if it has $d$ extrema in int $M$ (for $d=1 f$ is said to be unimodal). Let $f^{n}=f \circ f \circ \ldots \circ f$ denote the $n^{\text {th }}$ iterate of $f$.

By solenoid attractor of $M$ (or simply a solenoid) we mean a closed $f$-invariant subset $A \subset M$ of the following structure:

$$
A=\bigcap_{n=1}^{\infty} M^{(n)}, \quad M^{(1)} \supset M^{(2)} \supset \ldots,
$$

where

$$
M^{(n)}=\bigcup_{k=0}^{p_{n}-1} I_{k}^{(n)}
$$

is the union of $p_{n}$ closed disjoint intervals $I_{k}^{(n)}$ such that $f I_{k}^{(n)} \subset I_{k+1}^{(n)}$ (here $I_{p_{n}}^{(n)}$ is identified with $\left.I_{0}^{(n)}\right), p_{n} \rightarrow \infty$.

Clearly, $p_{n}$ is a divisor of $p_{n+1}$. The type of the solenoid $A$ is the maximal possible sequence $\left\{p_{n}\right\}_{n=1}^{\infty}$ of the pairwise distinct periods $p_{n}$.

Let $\lambda$ denote the Lebesgue measure on $M$ and $\operatorname{dim} X$ denote the Hausdorff dimension of a subset $X \subset M$. The aim of the present paper is to prove the following theorem: 
Theorem. Let $A$ be a solenoidal attractor of type $\left\{p_{n}\right\}_{n=0}^{\infty}$ of a map $f \in \mathfrak{A}$. Then 1) $\lambda(A)=0 ; 2)$ if $\sup \left(p_{n+1} / p_{n}\right)<\infty$ then $\operatorname{dim} A<1$.

For unimodal maps the theorem was proved earlier (see [G 2, BL 2, MMSS]). Our approach to multimodal maps is based upon the method developed in [L].

We refer to [B 1, B 2] for detailed study of topological properties of solenoids for continuous one dimensional maps. Now we state some of these properties.

An interval $J \subset M$ is called wandering if $f^{n} J \cap f^{m} J=\emptyset$ for $n>m \geqq 0$ and the orbit $\left\{f^{n} J\right\}_{n=0}^{\infty}$ doesn't tend to a limit cycle. It was proved recently that maps $f \in \mathfrak{A}$ have no wandering intervals. This result was obtained in [L, BL1] for maps $f \in \mathfrak{U}$ without inflection critical points. The restriction on critical points was removed in [MMS]. For injective and unimodal maps the absence of wandering intervals was earlier proved in [G1, Y, MS].

It follows from the absence of wandering intervals that any solenoidal attractor of $f \in \mathfrak{U}$ is nowhere dense (since an interval $J \subset \operatorname{int} A$ should be wandering). Consequently, the restriction $f \mid A$ is topologically conjugate to the transitive shift on a compact group [B1, B 2]. So, $f \mid A$ is a minimal dynamical system, i.e. there are no proper closed invariant subsets $A^{\prime} \subset A$. Hence, $A \cap B=\emptyset$ for any two distinct solenoids $A, B$.

Notice also that clearly $A$ has no isolated points and the restriction $f \mid A$ is injective.

Remarks. 1. We have proved that if $A$ is a non-periodic and non-solenoidal attractor in the sense of [M, BL 3] and $\lambda(A)>0$ then $f \mid A$ is essentially noninvertible. It follows that every absolutely continuous $f$-invariant measure has positive entropy. The proof will appear elsewhere.

2. Using the Milnor-Thurston kneading theory [MT] one can show that solenoids of all types occur even in the quadratic family $x \mapsto a x(1-x)$. The most popular of them is the so-called Feigenbaum attractor for which $p_{n}=2^{n}$. For this attractor one can prove the theorem using the renormalization group method [VSK].

3. The requirements of smoothness of $f$ in the theorem can be essentially weakened, as in the papers [BL1] or [MMS].

\section{Notations and Conventions}

If points $x$ and $y$ lie in the same component of $M,[x, y]$ denotes the (closed) interval ending at $x$ and $y$ (we don't assume $x \leqq y$ ). Similarly, if $L$ and $N$ are intervals, $[L, N]$

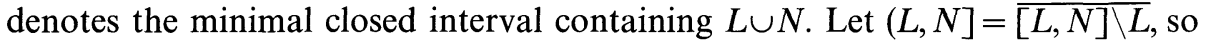
$(L, N]$ is closed when $L, N$ are intervals. by

At first let us introduce some notations and convenient conventions. Denote

$C=C(f)$ the set of critical points of $f, d=|C(f)|$;

$E=E(f)$ the set of extrema of $f$ lying in int $M$.

Replacing $M$ by some invariant submanifold $M^{(n)}$ of the definition (1)-(2), we can get $M$ to be the union of intervals and all critical points of $f$ to lie on the solenoid $A$ (just for this reason it is convenient to consider non-connected manifolds $M$ ): 
Besides, one can assume

$$
f(\partial M) \subset \partial M
$$

Indeed, let us include $M$ into a manifold $\tilde{M}$ with boundary so that $M \subset \operatorname{int} \tilde{M}$. Then one may construct a continuation $\tilde{f}: \tilde{M} \rightarrow \tilde{M}$ of $f$ such that $C(\tilde{f})=C(f)$ and $\tilde{f}(\partial M)$ $C \partial M$. Note that the property (3) is preserved.

It follows from (4) that all points of $\partial M$ are preperiodic (i.e. $f^{n} a=f^{n+p} a$ for some $n \geqq 0, p>0$ ), and hence $A \subset \operatorname{int} M$.

Further, replacing the smooth structure on $M$, we can reduce $f$ in neighbourhoods of critical points $c \in C(f)$ to the form

$$
f: x \mapsto f(c)+v(x-c)^{\kappa},
$$

where $v \in\{ \pm 1\}$ and $\kappa \in \mathbb{N}$ depend on $c$.

Let us define in neighbourhoods of extrema $c \in E(f)$ the involution $\tau: x \rightarrow x^{\prime}$ by the equation $f\left(x^{\prime}\right)=f(x)$. By (5) $\tau$ is just the reflection with respect to $c$.

Now, let us fix for the rest of the paper a small number $\eta>0$ such that $\eta$-neighbourhoods of points $c_{i} \in C(f) \cup \partial M$ are disjoint and (5) holds in $\eta$-neighbourhoods of critical points. Then the involution $\tau$ is well-defined in $\eta$-neighbourhoods of extrema.

Proposition 1. Let $f \in \mathfrak{U}, V$ be an interval whose orbit doesn't tend to a limit cycle. Then

$$
\inf _{m \in \mathbb{N}} \lambda\left(f^{m} V\right)>0
$$

Proof. Since $f \in \mathfrak{U}$ has no wandering intervals, $f^{p} V \cap V \neq \emptyset$ for some $p \in \mathbb{N}$. Hence, $I=\bigcup_{n=0}^{\infty} f^{p n} V$ is a periodic interval (perhaps non-closed). If $I$ contains two $f^{p}$-fixed points $\alpha, \beta$ then $f^{p n} V \supset\{\alpha, \beta\}$ for all sufficiently large $n$ and (6) follows. The simple analysis of the other case is left to the reader.

It follows from Proposition 1 that there exists $\xi>0$ such that

$$
\lambda(V) \geqq \eta \Rightarrow \lambda\left(f^{m} V\right)>\xi \quad(m=0,1, \ldots)
$$

for any interval $V \subset M$ containing some critical point. Fix such a $\xi$ from now on.

\section{Chains of Intervals}

A sequence of intervals $\mathbb{G}=\left\{G_{m}\right\}_{m=0}^{l}$ will be called a chain of intervals (of length $l$ ) if $f G_{m} \subset G_{m+1}$.

The multiplicity mult $\mathbb{G}$ of the chain is the maximal $\mu$ for which there exist $\mu$ intervals $G_{m}$ with a common point.

The order ord $\mathbb{G}$ of the chain is the number of intervals $G_{m}$ containing critical points. Clearly,

$$
\operatorname{ord} \mathbb{G} \leqq d \cdot \operatorname{mult} \mathbb{G}
$$

[recall that $d=|C(f)|$ is the number of critical points]. 
The chain $\mathbb{G}$ is called maximal if $G_{m}$ are maximal intervals for which $f G_{m} \subset G_{m+1}(m=0, \ldots, l-1)$. It is called cyclic if $f G_{l} \subset G_{0}$. When we say maximal cyclic chains, we mean $G_{l}$ also satisfies the maximality property.

The concept of maximal chain introduced in $[\mathrm{L}]$ is the effective tool in a number of problems of one dimensional dynamics. We use the convenient modification of the concept suggested in [MMS].

Suppose $G_{l}$ Cint $M, \lambda\left(G_{l}\right)<\xi \leqq \eta$, where $\xi$ and $\eta$ are defined in Sect. 2. Since $f(\partial M) \subset \partial M$ the intervals $G_{m}$ don't contain boundary points of $M(m=0,1, \ldots, l)$. Moreover, by (7) $\lambda\left(G_{m}\right)<\eta$ for any interval $G_{m}$ containing a critical point. Hence, $G_{m}$ can contain only one critical point.

Suppose additionally that $\mathbb{G}$ is maximal. Then $f\left(\partial G_{m}\right) \subset \partial G_{m+1}$. If $G_{m}$ contains an extremum $c$ then it is symmetric with respect to $c$. If $G_{m}$ doesn't contain extrema then $f G_{m}=G_{m+1}$.

In what follows we will consider only the chains satisfying $G_{l} \subset \operatorname{int} M$ and $\lambda\left(G_{l}\right)<\xi$.

For two chains $\mathbb{G}=\left\{G_{m}\right\}_{m=0}^{l}$ and $\mathbb{I}=\left\{I_{m}\right\}_{m=0}^{l}$ we write $\mathbb{G}>\mathbb{I}$ if $G_{m} \supset I_{m}$ $(m=0, \ldots, l)$. In such a case $G_{m}^{ \pm}$denotes the connected components of $G_{m} \backslash I_{m}$.

Given a chain $\mathbb{I}=\left\{I_{m}\right\}_{m=0}$ and an interval $G \supset I_{l}$, there exists the unique maximal chain $\mathbb{G}=\left\{G_{m}\right\}_{m=0}^{l}$ such that $\mathbb{G}>\mathbb{I}$ and $G_{l}=G$. Indeed, one may subsequently construct the intervals $G_{l}=G, G_{l-1}, \ldots, G_{0}$. We say that such a chain $\mathbb{G}$ is associated with the chain II and the last interval $G$.

Proposition 2. Let $\mathbb{I}=\left\{I_{m}\right\}_{m=0}^{p-1}$ be a cyclic chain of disjoint intervals, $\mathbb{I}_{l}=\left\{I_{m}\right\}_{m=0}^{l}$ be its subchain, $l \leqq p-1$. Consider another chain $\left\{G_{m}\right\}_{m=0}^{l}=\mathbb{G}>\mathbb{I}_{l}$. Suppose $G_{l}$ intersects $q$ intervals of the chain II. Then

a) mult $\mathbb{G} \leqq 2 q$;

b) $\operatorname{ord} \mathbb{G} \leqq 2 q d$.

Proof. Let mult $\mathbb{G}=\mu$. Then there exists a point $x$ belonging to $\mu$ intervals $G_{m_{1}}, \ldots, G_{m_{\mu}}$ of the chain $\mathbb{G}$. Clearly, one of these intervals, say $G_{m_{j}} \equiv G_{m}$, must intersect $\mu / 2$ intervals of the chain II. Since this chain is cyclic, the interval $G_{l} \supset f^{l-m} G_{m}$ also intersects at least $\mu / 2$ intervals of the chain II. Hence $\mu / 2 \leqq q$, and part a) is proved.

Part b) follows from a).

\section{Distortion Theorems Along Chains of Intervals}

In this section we sum up the information on the distortion of iterates $f^{l}$ of maps $f \in \mathfrak{A}$ uniform with respect to $l$.

All statements below have two versions: a) distortion of interval ratio and b) distortion of density of measurable set. Version b) is always stronger than a) but it is convenient to have both statements.

The first two lemmas concern $f$ itself.

Lemma 1a (cf. [L]). Let I be an interval divided into two subintervals $L$ and $R$. Then there is a constant $B$ such that, provided $\lambda(L) \leqq \lambda(R)$, we have

$$
\lambda(f L) / \lambda(f R) \leqq B \lambda(L) / \lambda(R)
$$

( $B$ is independent of $I, L$, and $R$ ). 
Remark. In the above and further lemmas all constants depend on $f$ but we don't notice this dependence.

Proof. Clearly, one can assume that $I$ lies in the $\eta$-neighbourhood of some critical point $c$. We restrict ourselves to the case when $c \in I$, since the other case is similar. Let $c$ divide $I$ into two intervals $I^{ \pm}=\left[a^{ \pm}, c\right]$, where for definiteness $\lambda\left(I^{+}\right) \geqq \lambda\left(I^{-}\right)$. It is clear from (5) that the case when $L$ ends at $a^{+}$is worse than the opposite case. So, assume $L$ ends at $a^{+}$and hence $R \ni c$ [since $\left.\lambda(L) \leqq \lambda(R)\right]$. Then by $(5) \lambda(f L)$ $=\left|f^{\prime}(x)\right| \lambda(L) \leqq \kappa \lambda(I)^{\kappa-1} \lambda(L)$ (here $x$ is an appropriate point of $L$ ) and $\lambda(f R$ ) $\geqq(\lambda(R) / 2)^{\kappa}$. Consequently,

$$
\frac{\lambda(f L)}{\lambda(f R)} \leqq \kappa 2^{\kappa}\left(\frac{\lambda(I)}{\lambda(R)}\right)^{\kappa-1} \frac{\lambda(L)}{\lambda(R)} \leqq \kappa 2^{2 \kappa-1} \frac{\lambda(L)}{\lambda(R)} .
$$

Lemma 1b. There is a constant $\beta$ such that, provided $\operatorname{dens}(X \mid I) \leqq 1 / 4$, we have

$$
\operatorname{dens}(f X \mid f I) \leqq \beta \operatorname{dens}(X \mid I) .
$$

Proof. Assume again that $I$ lies in the $\eta$-neighbourhood of a critical point $c$ which divides $I$ into the intervals $I^{ \pm}=\left[a^{ \pm}, c\right]$, and $\lambda\left(I^{+}\right) \geqq \lambda\left(I^{-}\right)$. Set $X^{ \pm}=X \cap I^{ \pm}$. Let us replace $X^{ \pm}$by the intervals $J^{ \pm} \subset I^{+}$ending at the same point $a^{+}$. Then by (5),

$$
\lambda\left(f J^{ \pm}\right) \geqq \lambda\left(f X^{ \pm}\right) \text {. }
$$

Set $\varepsilon=\operatorname{dens}(X \mid I)$. Then $\lambda\left(J^{ \pm}\right) / \lambda\left(I^{+}\right) \leqq 2 \varepsilon \leqq 1 / 2$ and applying Lemma 1 a we obtain

$$
\lambda\left(f J^{ \pm}\right) / \lambda\left(f I^{+}\right) \leqq 4 B \varepsilon \text {. }
$$

Finally, (8) and (9) imply

$$
\frac{\lambda(f X)}{\lambda(f I)} \leqq \frac{\lambda\left(f J^{+}\right)+\lambda\left(f J^{-}\right)}{\lambda\left(f I^{+}\right)} \leqq 8 B \varepsilon,
$$

as stated.

Lemma 2 (Koebe property [MS, S, BL1]). Let I and $K$ be two intervals, $I$ Cint $K, K^{ \pm}$ be components of $K \backslash I$. Suppose $K \cap C\left(f^{l}\right)=\emptyset$ (hence, $f^{l} / K$ is monotone). Then there are constants $\eta=\eta_{\mu}(\delta)>0$ and $H=H_{\mu}(\delta)$ such that provided mult $\left\{f^{m} K\right\}_{m=0}^{l} \leqq \mu$ and $\lambda\left(f^{l} K^{ \pm}\right) / \lambda\left(f^{l} I\right) \geqq \delta$, we have

a) $\lambda\left(K^{ \pm}\right) / \lambda(I) \geqq \eta$;

b) $\frac{\left|\left(f^{l}\right)^{\prime}(x)\right|}{\left|\left(f^{l}\right)^{\prime}(y)\right|} \leqq H$ for all $x, y \in I$.

The following two lemmas estimate the distortion of $f^{l}$ along the chain $\mathbb{G}$ of length $l$ depending only on mult $\mathbb{G}$.

Lemma 3a (cf. [L], Lemma 1.2). Let $\mathbb{G}=\left\{G_{m}\right\}_{m=0}^{l}$ be a maximal chain of intervals, $\left\{I_{m}\right\}_{m=0}^{l}=\mathbb{I} \prec \mathbb{G}$ be another chain. Let $\mu=$ mult $\mathbb{G}$. Then there exists a function $\omega=\omega_{\mu}(\delta)$ such that

$$
\frac{\lambda\left(G_{l}^{ \pm}\right)}{\lambda\left(I_{l}\right)} \geqq \delta \Rightarrow \frac{\lambda\left(G_{0}^{ \pm}\right)}{\lambda\left(I_{0}\right)} \geqq \omega
$$


Proof. Let us consider the maximal sequence of moments

$$
0=m(0)<m(1)<\ldots<m(s+1)=l
$$

such that $G_{m(i)} \cap C(f) \neq \emptyset$ for $i \in[1, s]$. Then

$$
s \leqq \operatorname{ord} \mathbb{G}+1 \leqq d \mu+1 .
$$

Denote by $c_{m(i)}$ the critical point belonging to $G_{m(i)} \cdot 1$

Assume for some $i \in[1, s+1]$,

$$
\lambda\left(G_{m(i)}^{ \pm}\right) / \lambda\left(I_{m(i)}\right) \geqq \delta_{i} .
$$

Then we will show

$$
\lambda\left(G_{m(i-1)}^{ \pm}\right) / \lambda\left(I_{m(i-1)}\right) \geqq \omega\left(\delta_{i}\right)=\delta_{i+1} .
$$

Iterating $(s+1)$ times, we will obtain the required estimate for $\lambda\left(G_{0}^{ \pm}\right) / \lambda\left(I_{0}\right)$.

The method of proving $(11) \Rightarrow(12)$ consists of two steps: at first we use the Koebe property of $f^{m(i)-m(i-1)-1} \mid G_{m(i-1)+1}$ (Lemma 2a) and then apply Lemma 1a to $f \mid G_{m(i-1)}$. At the first step we get

$$
\lambda\left(G_{m(i-1)+1}^{ \pm}\right) / \lambda\left(I_{m(i-1)+1}\right) \geqq \eta_{\mu}\left(\delta_{i}\right) .
$$

Note that we may use the Koebe property since the map $f^{m(i)-m(i-1)-1}$ : $G_{m(i-1)+1} \rightarrow G_{m(i)}$ has no critical points and is surjective due to maximality of the chain $\mathbb{G}$.

To make the second step, set $c=c_{m(i-1)}$ and consider several cases.

(i) $c \in C(f) \backslash E(f)$ is an inflection point. Then by Lemma 1a,

$$
\lambda\left(G_{m(i-1)}^{ \pm}\right) / \lambda\left(I_{m(i-1)}\right) \geqq B^{-1} \cdot \eta_{\mu}\left(\delta_{i}\right) .
$$

(ii) $c \in E(f)$ is an extremum and $c \in I_{m(i-1)}$. Let $c$ divide $I_{m(i-1)}$ into the intervals $I_{m(i-1)}^{ \pm}$. Suppose, to be definite, $\lambda\left(I_{m(i-1)}^{+}\right) \geqq \lambda\left(I_{m(i-1)}^{-}\right)$. Since $G_{m(i-1)}$ is symmetric with respect to $c$, we have by Lemma $1 \mathrm{a}$,

$$
\lambda\left(G_{m(i-1)}^{ \pm}\right) / \lambda\left(I_{m(i-1)}\right) \geqq \frac{1}{2} \lambda\left(G_{m(i-1)}^{+}\right) / \lambda\left(I_{m(i-1)}^{+}\right) \geqq \frac{1}{2} B^{-1} \cdot \eta_{\mu}\left(\delta_{i}\right) .
$$

(iii) $c \in E(f)$ and $c \notin I_{m(i-1)}$. To be definite, suppose $c \in G_{m(i-1)}^{+}$. Then by Lemma 1a,

$$
\lambda\left(G_{m(i-1)}^{-}\right) / \lambda\left(I_{m(i-1)}\right) \geqq B^{-1} \cdot \eta_{\mu}\left(\delta_{i}\right) .
$$

But as $G_{m(i-1)}$ is symmetric, $\lambda\left(G_{m(i-1)}^{+}\right) \geqq \lambda\left(G_{m(i-1)}^{-}\right)$. So, (12) holds in all cases.

The lemma is proved.

Lemma 3b. Let $X \subset M$ be a measurable set. Under the assumptions of Lemma $3 a$ provided $f I_{m}=I_{m+1}(0 \leqq m<l)$, there exists a function $\Omega=\Omega_{\mu, \delta}(\varepsilon)$ such that $\Omega(\varepsilon) \rightarrow 0$ $(\varepsilon \rightarrow 0)$ and

$$
\left.\begin{array}{l}
\lambda\left(G_{l}^{ \pm}\right) / \lambda\left(I_{l}\right) \geqq \delta \\
\operatorname{dens}\left(X \mid I_{0}\right) \leqq \varepsilon
\end{array}\right\} \Rightarrow \operatorname{dens}\left(f^{l} X \mid I_{l}\right) \leqq \Omega(\varepsilon)
$$

\footnotetext{
${ }^{1}$ Recall that we assume throughout the paper that $\lambda\left(G_{l}\right)<\xi$ and hence $G_{m(i)}$ contains at most one critical point (see Sect. 3)
} 
Proof. The proof of this lemma is analogous to that of Lemma 3a, but one should use Lemmas 1b, 2b instead of Lemmas 1a, 2a.

More precisely, considering as in Lemma 3 a the moments $m(i)$ for which $G_{m(i)}$ covers some critical point $c_{i}$, we must show that

$$
\operatorname{dens}\left(f^{m(i-1)} X \mid I_{m(i-1)}\right) \leqq \varepsilon_{i-1} \Rightarrow \operatorname{dens}\left(f^{m(i)} X \mid I_{m(i)}\right) \leqq \varepsilon_{i},
$$

where $\varepsilon_{i}$ depends on $\varepsilon_{i-1}$ only. To this end we estimate the distortion of

$$
f^{m(i)-m(i-1)} \mid I_{m(i-1)}
$$

in two steps.

In the first step we obtain by Lemma 1a that

$$
\operatorname{dens}\left(f^{m(i-1)+1} X \mid I_{m(i-1)+1}\right) \leqq \beta \varepsilon_{i-1} .
$$

In the second step we use the Koebe property to estimate the distortion of the diffeomorphism

$$
f^{m(i)-m(i-1)-1}: G_{m(i-1)+1} \rightarrow G_{m(i)} .
$$

It is possible since by Lemma $3 \mathrm{a} \lambda\left(G_{m(i)}^{ \pm}\right) / \lambda\left(J_{m(i)}\right) \geqq \omega=\omega_{\mu}(\delta)$. Consequently,

$$
\operatorname{dens}\left(f^{m(i)} X \mid I_{m(i)}\right) \leqq H \beta \varepsilon_{i-1},
$$

where $H=H_{\mu}(\omega)$ is the constant from Lemma $2 \mathrm{~b}$. So, we have obtained the required.

Note that on both steps the surjectivity of $f: I_{m(j)} \rightarrow I_{m(j+1)}$ is essential.

\section{The Ratio of Generating Intervals}

Let $\mathbb{I}^{(n)}=\left\{I_{m}^{(n)}\right\}_{m=0}^{p_{n}-1}$ denote the cyclic chains of intervals generating the solenoid $A$ [see definitions (1)-(2)]. Clearly, we can choose intervals $I_{0}^{(n)}$ in such a way that the map $f^{p_{n}}: I_{0}^{(n)} \rightarrow I_{0}^{(n)}$ is surjective. Set $I_{k}^{(n)}=f^{k} I_{0}^{(n)}\left(k=1, \ldots, p_{n}-1\right)$. Then

$$
f^{m} I_{k}^{(n)}=I_{k+m}^{(n)}
$$

where $k+m$ is considered $\bmod p_{n}$.

We also need the other family of cyclic chains $\widetilde{I}^{(n)}=\left\{\widetilde{I}_{m}^{(n)}\right\}_{m=0}^{p_{n}-1}$ generating $A_{n}$. The $\widetilde{\mathbb{I}}^{(n)}$ is defined as the cyclic chain such that $\widetilde{\mathbb{I}}^{(n)}>\mathbb{I}^{(n)}$,

$$
\widetilde{I}^{(n)}=I_{m}^{(n)} \cup \tau\left(I_{m}^{(n)}\right)
$$

if $I_{m}^{(n)}$ contains an extremum, and $f \widetilde{I}_{m}^{(n)}=\widetilde{I}_{m+1}^{(n)}$ otherwise. It is easy to check the existence of such a unique chain. Set

$$
\tilde{M}^{(n)}=\bigcup_{m=0}^{p_{n}-1} \widetilde{I}_{m}^{(n)}
$$

Since the restriction of $f$ onto the solenoid $\bigcap_{n=1}^{\infty} \tilde{M}^{(n)}$ is a minimal dynamical system, we have $\bigcap_{n=1}^{\infty} \tilde{M}_{n}=A$. 
Lemma 4. Consider an interval $\tilde{I} \in \widetilde{\mathbb{I}}^{(n)}$ containing an extremum and the nearest to $\tilde{I}$ interval $J \in \mathbb{I}^{(n)}$. Then

$$
\frac{\lambda(\widetilde{I}, J]}{\lambda(\widetilde{I})} \geqq \delta>0,
$$

where $\delta$ is independent of $n, I, J$.

Remark 1. In fact, the proof shows that $\lambda(I, J] / \lambda(I) \geqq \delta>0$ for any $I, J \in \mathbb{I}^{(n)}$.

Proof. Let $\tilde{I}$ be the symmetrization of $I \in \mathbb{I}^{(n)}$. Index the intervals $I_{m}^{(n)}$ in such a way that $I=I_{0}^{(n)}$ (and of course $\left.f I_{m}^{(n)}=I_{m+1}^{(n)}\right)$.

Fix an extremum $c \in A$. Since $A$ has no isolated points, there exist one or two points $x_{v} \in A \cap(c, c+v \xi)$ for $v \in\{ \pm 1\}$. Let

$$
\alpha=\min _{v}\left(\left|x_{v}-c\right|,\left|c+v \xi-x_{v}\right|\right) .
$$

Since $A$ is nowhere dense,

$$
\sup _{m} \lambda\left(I_{m}^{(n)}\right) \rightarrow 0 \quad(n \rightarrow \infty) .
$$

Let $n$ be so large that

$$
\sup _{m} \lambda\left(I_{m}^{(n)}\right)<\alpha / 2
$$

Then the interval $I_{s}^{(n)}$ containing $c$ doesn't cover $x_{v}$, and the intervals $I_{m(v)}^{(n)}$ containing $x_{v}$ lie in $[c-\xi, c+\xi], m(v) \neq s$. Let $L_{0}=I_{s}^{(n)} \cup \tau\left(I_{s}^{(n)}\right)$ be the symmetrization of $I_{s}^{(n)}$.

Let $L_{1} \subset[c-\xi, c+\xi]$ be the nearest to $L_{0}$ interval of $\mathbb{I}^{(n)}$. To be definite, assume that $L_{1}$ lies to the right of $L_{0}$. Consider then all intervals $L_{1}, \ldots, L_{k}$ of the chain $\mathbb{I}^{(n)}$ lying in $(c, c+\xi]$ indexed from left to right.

Since $\left[L_{0}, L_{k}\right] \supset\left[c, x_{+}\right] \supset[c, c+\alpha]$ we have

$$
\lambda\left(L_{0}\right)+\sum_{i=0}^{k-1} \lambda\left(L_{i}, L_{i+1}\right] \geqq \alpha .
$$

As $\lambda\left(L_{0}\right)<\alpha / 2$ there is an $i \in[0, k-1]$ such that

$$
\lambda\left(L_{i}, L_{i+1}\right] \geqq \frac{1}{2} \lambda\left(L_{i}\right) .
$$

Consider such a minimal $i$. Let $L_{-1}=\tau\left(L_{1}\right)$. Consider the interval $G=\left[\alpha_{i-1}, \alpha_{i+1}\right]$, where $\alpha_{j}$ denote the midpoints of $L_{j}$. Let $G^{ \pm}$be the right-hand and left-hand components of $G \backslash L_{i}$ correspondingly. Then by (14),

$$
\begin{gathered}
\frac{\lambda\left(G^{+}\right)}{\lambda\left(L_{i}\right)} \geqq \frac{1}{2} \cdot \frac{\lambda\left(L_{i}, L_{i+1}\right]}{\lambda\left(L_{i}\right)} \geqq \frac{1}{4}, \\
\frac{\lambda\left(G^{-}\right)}{\lambda\left(L_{i}\right)} \geqq \frac{1}{2} \frac{\lambda\left(L_{i-1}\right)}{\lambda\left(L_{i}\right)} \geqq 1 \quad(i>0) .
\end{gathered}
$$

If $i=0$ then $G$ and $L_{i}$ are symmetric with respect to $c$, so

$$
\frac{\lambda\left(G^{-}\right)}{\lambda\left(L_{i}\right)}=\frac{\lambda\left(G^{+}\right)}{\lambda\left(L_{0}\right)} \geqq \frac{1}{4} \quad(i=0) \text {. }
$$


Further, let $L_{i}=I_{l}^{(n)}=f^{l} I, 0 \leqq l<p_{n}$. Let us construct the maximal chain of intervals $\mathbb{G}=\left\{G_{m}\right\}_{m=0}^{l}$ such that $G_{l}=G$ and $G_{m} \supset I_{m}^{(n)}$ for $m=0,1, \ldots, l$. Since $G_{l}$ intersects at most three intervals of the chain $\mathbb{I}^{(n)}$ we get by Proposition 2

$$
\text { mult } \mathbb{G} \leqq 6 .
$$

Now make use of Lemma 3a taking in account (15)-(17) (and replacing $I$ by $\widetilde{I}$ ):

$$
\frac{\lambda\left(G_{0}^{ \pm}\right)}{\lambda(\widetilde{I})} \geqq \omega_{6}\left(\frac{1}{4}\right) \equiv \delta .
$$

Since $G_{l}$ contains the unique interval $L_{i}=I_{l}^{(n)}$ of the cyclic chain $\mathbb{I}^{(n)}$, the property (13) implies that $G_{0} \subset[J, \tau(J)]$, and we are done.

Lemma 5. There exists $\varrho<1$ such that for any $n \in \mathbb{N}, m \in\left[0, p_{n}-1\right]$ the following estimate holds:

$$
\operatorname{dens}\left(\tilde{M}^{(n+1)} \mid \widetilde{I}_{m}^{(n)}\right) \leqq \varrho .
$$

Proof. Denote $\tilde{K}=\widetilde{I}_{m}^{(n)}$. Let us consider the least $l \in\left[0, p_{n}-1\right]$ for which the interval $\widetilde{K}_{l}=f^{l} \widetilde{K}$ covers some extremum $c$. Note that $\widetilde{K}_{l} \in \tilde{\mathbb{I}}^{(n)}$ is symmetric with respect to $c$ and $f^{l}: \widetilde{K} \rightarrow \widetilde{K}_{l}$ is a diffeomorphism. First we estimate the density of $\tilde{M}^{(n+1)}$ in $\widetilde{K}_{l}$ and then pull it back to $\widetilde{K}$.

Consider the interval $\tilde{I} \in \tilde{\mathbb{I}}^{(n+1)}$ containing $c, \tilde{I} \subset \widetilde{K}_{l}$. Let $J \in \mathbb{I}^{(n+1)}$ be the nearest to $\tilde{I}$ interval. Clearly, $J \cup \tau(J) \subset \widetilde{K}_{l}$. So, Lemma 4 yields

$$
\lambda\left(\tilde{K}_{l} \backslash \widetilde{I}\right) \geqq 2 \lambda(\tilde{I}, J] \geqq 2 \delta \cdot \lambda(\widetilde{I}),
$$

hence,

$$
\lambda\left(\tilde{K}_{l} \backslash \tilde{I}\right) \geqq 2 \gamma \lambda\left(\tilde{K}_{l}\right)
$$

where $\gamma=\delta /(2 \delta+1)$.

Let $Z=\tilde{M}^{(n+1)} \cap\left(\tilde{K}_{l} \backslash \widetilde{I}\right)$. Since $f \mid Z$ is injective, $Z \cap \tau(Z)=\phi$. Hence

$$
\lambda(Z) \leqq \frac{1}{2} \lambda\left(\tilde{K}_{l} \backslash \tilde{I}\right) .
$$

This estimate and (18) imply

$$
\lambda\left(\tilde{M}^{(n+1)} \cap K_{l}\right)=\lambda(\widetilde{I})+\lambda(Z) \leqq \lambda\left(\widetilde{K}_{l}\right)-\frac{1}{2} \lambda\left(\tilde{K}_{l} \backslash \widetilde{I}\right) \leqq(1-\gamma) \lambda\left(\widetilde{K}_{l}\right) .
$$

Further, let $N$ be the nearest to $\widetilde{K}_{l}$ interval of the chain $\mathbb{I}^{(n)}$. Let us construct the maximal chain of intervals $\mathbb{G}=\left\{G_{r}\right\}_{r=0}^{l}$ such that $G_{l}=[N, \tau(N)]$ and $G_{r} \supset \widetilde{K}_{r}$ $(r=0,1, \ldots, l)$. By Lemma 4 ,

$$
\frac{\lambda\left(G_{l}^{ \pm}\right)}{\lambda\left(\widetilde{K}_{l}\right)}=\frac{\lambda\left(\tilde{K}_{l}, N\right]}{\lambda\left(\widetilde{K}_{l}\right)} \geqq \delta .
$$

By Proposition 2

$$
\text { mult } \mathbb{G} \leqq 4 .
$$

Denote $X=\tilde{K} \backslash \tilde{M}^{(n+1)}$, dens $(X \backslash \widetilde{K})=\varepsilon$. Lemma $3 b$ with (20) and (21) yield

$$
\operatorname{dens}\left(f^{l} X \mid \tilde{K}_{l}\right) \leqq \Omega(\varepsilon) \equiv \Omega_{4, \delta}(\varepsilon) \text {. }
$$


Hence dens $\left(\tilde{M}^{(n+1)} \mid \tilde{K}_{l}\right) \geqq 1-\Omega(\varepsilon)$. Comparing this with (19) we conclude $\Omega(\varepsilon) \geqq \gamma$. Thus, $\varepsilon \geqq \varepsilon_{0}>0$, where $\varepsilon_{0}$ depends on $f$ only. Finally, set $\varrho=1-\varepsilon_{0}$.

Remark. Using the above argument, one can show that $\lambda\left(\widetilde{I}_{m}^{(n)}\right) \leqq C \cdot \lambda\left(I_{m}^{(n)}\right)$.

\section{Proof of the Theorem}

It follows from Lemma 5 that

$$
\lambda\left(\tilde{M}^{(n+1)}\right) \leqq \varrho \cdot \lambda\left(\tilde{M}^{(n)}\right) .
$$

This implies the first part of the theorem:

$$
\lambda(A)=\lim _{n \rightarrow \infty} \lambda\left(\tilde{M}^{(n)}\right)=0 .
$$

Now let $N=\max _{n \in \mathbb{N}}\left(p_{n+1} / p_{n}\right)<\infty$. Find such an index $q \in(0,1)$ that for all numbers $x_{i} \in[0,1](i=1, \ldots, N)$ the following property holds

$$
\sum x_{i} \leqq \varrho<1 \Rightarrow \sum x_{i}^{q} \leqq 1 \text {. }
$$

Let $\tilde{I}_{0}^{(1)} \supset \widetilde{I}_{0}^{(2)} \supset \ldots$ and $\varphi_{n}: \mathbb{Z}_{p_{n+1}} \rightarrow \mathbb{Z}_{p_{n}}$ be the natural homomorphism. So, $\widetilde{I}_{m}^{(n+1)} \subset \widetilde{I}_{k}^{(n)}$ iff $\varphi_{n}(m)=k$.

By Lemma 5 for any $k \in\left[0, p_{n}-1\right]$ we have

$$
\sum_{m: \varphi_{n}(m)=k} \lambda\left(\widetilde{I}_{m}^{(n+1)}\right) \leqq \varrho \lambda\left(\widetilde{I}_{k}^{(n)}\right) .
$$

Consequently

$$
\sum_{m: \varphi_{n}(m)=k} \lambda\left(\widetilde{I}_{m}^{(n+1)}\right)^{q} \leqq \lambda\left(\tilde{I}_{k}^{(n)}\right)^{q}
$$

Summing by $k \in[0, q-1]$ yields

$$
\sum_{m=0}^{p_{n+1}-1} \lambda\left(I_{m}^{(n+1)}\right)^{q} \leqq \sum_{k=0}^{p_{n}-1} \lambda\left(I_{k}^{(n)}\right)^{q}
$$
Thus

Consequently, $l_{q}(A)<\infty$, where $l_{q}$ denotes $q$-dimensional Hausdorff measure.

$$
\operatorname{dim} A \leqq q,
$$

and the theorem is completely proved.

\section{References}

[B 1] Blokh, A.M.: Decomposition of dynamical systems on an interval. Russ. Math. Surv. 38; N. 5, 133-134 (1983) [see also Blokh, A.M.: Letter to the editors. Russ. Math. Surv. 42, (1987)]

[B 2] Blokh, A.M.: On dynamical systems on one dimensional branched manifolds (in Russian), I, II, III; Theory Funct. Functional Anal. Appl. 46, 8-18 (1986); 47, 67-77 (1987); 48, 32-46 (1987)

[BL 1] Blokh, A.M., Lyubich, M.Yu.: Non-existence of wandering intervals and structure of topological attractors of one dimensional dynamical systems. II. The Smooth case, preprint (1987) 
[BL 2] Blokh, A.M., Lyubich, M.Yu.: Measure of solenoidal attractors of unimodal maps of an interval (1987). Matem. Zametki (to appear)

[BL 3] Blokh, A.M., Lyubich, M.Yu.: Attractors of maps of the interval. Funct. Anal. Appl.21, 70-71 (1987)

[G1] Guckenheimer, J.: Sensitive dependence on initial conditions for one dimensional maps. Commun. Math. Phys. 70, 133-160 (1979)

[G 2] Guckenheimer, J.: Limit sets of $S$-unimodal maps with zero entropy. Commun. Math. Phys. 110, 655-659 (1987)

[L] Lyubich, M.Yu.: Non-existence of wandering intervals and structure of topological attractors of one dimensional dynamical systems. I. The case of negative Schwarzian derivative, preprint (1987)

[MS] De Melo, W., van Strien, S.J.: A structure theorem in one dimensional dynamics. Preprint (1986)

[MMS] Martens, M., de Melo, W., van Strien, S.J.: Julia-Fatou-Sullivan theory for real onedimensional dynamics. Preprint (1988)

[MMSS] Martens, M., de Melo, W., van Strien, S.J., Sullivan, D.: Bounded geometry and measure of the attracting Cantor set of quadratic-like maps, preprint (1988)

[M] Milnor, J.: On the concept of attractor. Commun. Math. Phys. 99, 177-195 (1985)

[MT] Milnor, J., Thurston, W.: On iterated maps of the interval. I, II, preprint (1977)

[S] Van Strien, S.: Hyperbolicity and invariant measures for general $C^{2}$-interval maps satisfying Misiurewicz condition. Preprint Delft University of Technology, pp. 27-46

[Y] Yoccoz, J.-C.: Il n'y a pas de contre-exemples de Denjoy analytiques. C.R. Acad. Sci. Paris, 289, 141-144 (1984)

[VSK] Vul, E.B., Sinai, Ya.G., Khanin, K.M.: Universality of Feigenbaum and thermodynamical formalism. Russ. Math. Surv. 39, 3-37 (1984)

Communicated by Ya. G. Sinai

Received December 1, 1988; in revised form March 15, 1989 
